\title{
Effective mass and dielectric constant mismatch effects in spherical multishell quantum dots
}

\author{
M. Royo and J. Planelles \\ Departament de Ciències Experimentals, Universitat Jaume I, Box 224, E-12080 Castelló, Spain \\ M. Pi \\ Departament ECM, Facultat de Física and $I N^{2}$ UB, Universitat de Barcelona, E-08028 Barcelona, Spain
}

(Received 21 September 2006; published 9 January 2007)

\begin{abstract}
The role of effective mass and dielectric mismatches on chemical potentials and addition energies of manyelectron multishell quantum dots (QDs) is explored within the framework of a recent extension of the spin density functional theory. It is shown that although the gross electronic density is located in the wells of these multishell QDs, taking position-dependent effective mass and dielectric constant into account can lead to the appearance of relevant differences in chemical potential and addition energies as compared to standard calculations in which the effective mass and the dielectric constant of the well is assumed for the whole multishell structure.
\end{abstract}

DOI: 10.1103/PhysRevB.75.033302

PACS number(s): 73.21.La, 71.15.Mb

Over a decade ago, Eychmüller and coworkers ${ }^{1,2}$ opened the door to the nano-heterostructures called quantum dot quantum wells (QDQW). These primal spherical multishell nanostructures were composed of a CdS central core surrounded by an $\mathrm{HgS}$ shell, a little thicker than a monolayer, capped with additional CdS. The synthesis route was based on the chemical control of the composition of the shells as the structure was grown layer by layer, so that the thickness of the core, well, and clad can be varied during the course of the synthesis. The basic physics of QDQWs derives from the band gap differences between neighboring materials leading to a radial confinement profile for carriers both in the conduction and in valence bands. Thus, in a CdS QD with an $\mathrm{HgS}$ quantum well inside, the electronic density, coming from either optical excitations or electronic levels charging, is localized in the $\mathrm{HgS}$ well. ${ }^{3}$ The relatively easy control of the size and composition of these structures and, therefore, the possibility of modifying the charge density localization, grants a high degree of flexibility for tailoring the discrete energy spectra of these systems. ${ }^{4}$ More recently, Al-Sayed and coworkers, ${ }^{5}$ by means of alternative precipitation of $\mathrm{HgS}$ and $\mathrm{CdS}$ on a CdS central core, successfully obtained spherical nanoparticles containing two $\mathrm{HgS}$ quantum wells separated by a double CdS barrier. This work, together with other multishell synthesis reported in recent years, ${ }^{6,7}$ prompt multishell nanostructures as firm candidates to become versatile components of electronic devices.

Theoretical studies on spherical multishell systems at the monoelectronic or excitonic level employing atomistic tightbinding models ${ }^{8}$ or macroscopic-like methods, such as the one-band effective mass approach ${ }^{9,10}$ and the more accurate multiband approaches, ${ }^{11}$ have been reported. The influence of a magnetic field on the electron and hole energy spectra of multishell QDs has also been been reported. ${ }^{12}$ Nevertheless, not many works on charging electrons in spherical multishell quantum dots have been carried out. We mention the work by Banin et al., ${ }^{13,14}$ who studied an electronically charged core/ shell InAs/ZnSe spherical nanocrystal spectroscopically. Some of the features observed in this heterostructure were theoretically reproduced by Dai et al. ${ }^{15}$ using unrestricted Hartree-Fock-Roothaan calculations, including up to nine electrons.
In a recent paper, ${ }^{16}$ we developed a method, within the framework of the spin density functional theory (SDFT), capable of studying many-electron spherical QDs including effective mass and dielectric mismatches. In a multishell QD, an electron feels different effective masses and dielectric constants in the different layers. In systems composed of $\mathrm{CdS}$ and $\mathrm{HgS}$, the electronic density is mainly concentrated in the HgS wells. This fact might lead one to think that it is safe to employ the effective mass and dielectric constant of the $\mathrm{HgS}$ for the whole multishell QD. In the present work we will show that a correct description of such systems requires consideration of a proper variable, i.e. position-dependent, effective mass, and dielectric constant. SDFT results, which employ the effective mass and dielectric constant of the well for the whole QD (hereafter referred to as unpolarized calculation) and those with position-dependent parameters (polarized calculation), show differences in chemical potential. This leads to differences in the number of electrons coming into the $\mathrm{HgS}$ well and also differences in addition energies, in some cases reflecting distinct ground state electronic configurations in either the polarized or the unpolarized case. Differences increase with the number of layers of the multishell QD.

A fully detailed description of the method employed can be found in Ref. 16. In short, it is an extension of the SDFT, ${ }^{18}$ which includes (i) position-dependent effective mass by replacing the standard kinetic energy operator $-\frac{\hbar^{2}}{2 m^{*}} \nabla^{2}$ by $-\frac{\hbar^{2}}{2} \nabla\left(\frac{1}{m^{*}} \nabla\right)$, corresponding to the case of variable effective mass, (ii) polarization of the Coulomb interaction arising from the dielectric mismatch, by numerically integrating the Poisson equation $\nabla[\varepsilon(r) \nabla \Psi(r)]=-4 \pi n(r)$; (iii) Self-energy, also coming from the dielectric mismatch, by incorporating the mono-electronic self-polarization potential according to the equations reported in Ref. 19. This dielectric confinement and the spatial confinement potentials are the genuine single-particle components of the Konh-Sham potential; (iv) the effect of dielectric mismatch on exchange by means an appropriate scaling of the exchange functional, which is consistent with the Coulomb functional employed; and finally, (v) the correlation functional is also modified to incorporate the position-dependent parameters properly by 
means of a consistent scaling of the Perdew-Zunger analytical functional employed. ${ }^{20}$

The material parameters employed in our calculations, namely electron effective masses $m_{\mathrm{HgS}}^{*}=0.04, m_{\mathrm{CdS}}^{*}=0.15$, $m_{\mathrm{H}_{2} \mathrm{O}}^{*}=1$, dielectric constants $\varepsilon_{\mathrm{HgS}}=11.4, \varepsilon_{\mathrm{CdS}}=5.5, \varepsilon_{\mathrm{H}_{2} \mathrm{O}}$ $=1.78$, and band offsets $V_{\mathrm{HgS} / \mathrm{CdS}}=1.35 \mathrm{eV}, \quad V_{\mathrm{HgS} / \mathrm{H}_{2} \mathrm{O}}$ $=4.15 \mathrm{eV}$, and $V_{\mathrm{CdS} / \mathrm{H}_{2} \mathrm{O}}=2.8 \mathrm{eV}$ are taken from Refs. 10 and 17. The bottom of the $\mathrm{HgS}$ conduction band is assumed to be the origin of energies. We have carried out calculations from one up to 25 electrons, the orbital basis set employed including orbitals $1 s, 1 p, 1 d, 1 f, 2 s$, and $2 p$.

Figure 1(a) shows the calculated chemical potential ${ }^{21}$ vs the number of electrons of one of the multishell QDs synthesized by Al-Sayed et al. ${ }^{5}$ The heterostructure is represented in the inset. It is built of an internal CdS core of radius $3.2 \mathrm{~nm}$, a middle well-acting $\mathrm{HgS}$ shell with a thickness of $0.8 \mathrm{~nm}$ and an external $0.4 \mathrm{~nm}$ thick CdS clad. The QD is surrounded by water.

Chemical potentials obtained from the polarized calculations, either including self-energy or not, indicate that up to two electrons can come into the $\mathrm{HgS}$ well, while unpolarized calculations already yield a chemical potential for the singleelectron QD exceeding the $\mathrm{HgS} / \mathrm{CdS}$ confining barrier. The two horizontal lines in Fig. 1 at 1.35 and $4.15 \mathrm{eV}$ represent the confining barrier height of the $\mathrm{HgS}$ well and the QD, respectively. Therefore, Fig 1(a) indicates that while the polarized calculations predict that the QD can accept up to a maximum of 16 electrons, the limit of electrons that can come into the QD yielded by the unpolarized calculations exceeds 25. Concerning addition energies [see Fig. 1(f)], no qualitative differences can be seen between the two approaches employed. The profiles obtained indicate the fulfillment of the Afbau and Hund rules, the same spin and electronic configurations being achieved by all approaches. As a consequence, no appreciable quantitative differences are found when the single-particle self-energy is included, as it almost cancels completely out. Only quantitative differences between polarized and unpolarized calculations can be seen that reveal the extra polarization work.

Since, as pointed out above, it is relatively easy to control the layer thickness in a multishell QD experimentally, we then calculate several multishell structures, including one or more $\mathrm{HgS}$ wells separated by $\mathrm{CdS}$ barriers of different widths in order to illustrate the relevance of including position-dependent effective mass and dielectric constant in the calculation of chemical potentials and additions energies. The notation $A / B / A / \ldots x / y / z / \ldots$ that will be employed hereafter means a QD built of an $x \mathrm{~nm}$ radius internal core of material $A$ covered by successive $y, z, \ldots \mathrm{nm}$ thickness shells of materials $B / A / \ldots$. In all cases the medium surrounding the QD is water.

Figure 1(b) and 1(g) correspond to CdS/HgS/CdS 3/2/1 multishell QD. The same general trends as in the previous case are found here. Namely, the same spin and electronic configuration are achieved by all approaches, all of them yielding qualitatively similar addition energies, these revealing the fulfillment of the Afbau and Hund rules, but quantitative differences related to the polarization work. Finally, relevant differences in chemical potentials between the po- larized and unpolarized approach are found, the self-energy playing a minor role.

We deal next with double quantum wells. We first consider $\mathrm{HgS} / \mathrm{CdS} / \mathrm{HgS} / \mathrm{CdS} 3.5 / 3 / 2 / 1$ QD, whose wellacting core is separated from the second well by a barrier, the last shell also being barrier-acting [see Figs. 1(c) and 1(h)]. As above, the main differences between polarized and unpolarized approaches can be found when calculating chemical potentials. Thus, while the unpolarized calculation predicts that all 25 electrons come into the $\mathrm{HgS}$ wells, the polarized calculation already does not allow the 11th electron to come into the wells, as the chemical potential of this QD populated with 11 electrons exceeds the well-confining potential height. The addition energy profile reveals a peculiar change of maxima positions with respect to the other QDs that have been studied. This is the outcome of a different orbital sequential filling $1 s, 2 s, 1 p, 1 d \ldots$ (instead of $1 s, 1 p, 1 d \ldots$ ) which has its origins in a relative stabilization of the $2 s$ orbital that locates its radial node in the barrier region. Regarding differences between polarized and unpolarized calculations, a first qualitative difference between them appears at $E_{\text {add }}(2)$, which is not a maximum if polarization is included, this fact being related to the extra stability of the $2 s$ orbital originated by polarization.

The last two structures we consider are QDs with double quantum wells having a barrier-acting core $(\mathrm{CdS} / \mathrm{HgS} / \mathrm{CdS} / \mathrm{HgS} / \mathrm{CdS})$, the only difference between them being the thickness of the intermediate $\mathrm{CdS}$ barrier. Figure 1(d) and 1(i) show the result for $3 / 2 / 1 / 2 / 1$ while Fig. 1(e) and 1(j) correspond to $3 / 2 / 2 / 2 / 1$. Besides the discrepancies in chemical potential between polarized and unpolarized calculations, qualitatively similar to the previously outline for the other QDs studied, now differences arise in the addition energy plot. In the case of the thinner CdS barrier, Fig. 1(i), the third big maximum appears at a different number of electrons $N$ in the unpolarized and polarized case, which is the result of a different sequential filling, $1 s^{2}, 1 p^{6}, 1 d^{10}, 1 f^{6}$, vs $1 s^{2}, 1 p^{6}, 2 s^{2}, 1 d^{10}, 1 f^{4}$, originated by an extra stabilization of the $2 s$ orbital coming from polarization. It should also be mentioned that self-energy gives rise to a few ground-state reconstructions that are reflected in small changes in the addition energy profile, even leading to a spin change $\left(2 S_{z}=6\right.$ vs 4$)$ at $N=14$.

The most relevant role of both polarization and selfenergy can be seen in the case of the thicker CdS barrier. Thus, Fig. 1(j) reveals that, in the region of few electrons, a quite different $E_{\text {add }}$ vs $N$ profile results when polarization is included, this profile undergoing a further relevant change as the self-energy is accounted for. We can mention, for example, that at $N=8$ electrons, the spin predicted by the unpolarized calculation is $2 S_{z}=0$, which turns into $2 S_{z}=2$ as polarization is included, and recovers a zero value as, additionally, self-energy is incorporated.

In short, we have shown that despite the fact that the electron density may be mainly concentrated in the wells of a multishell QD, position-dependent effective mass and dielectric constant should be employed for a proper calculation of chemical potentials and addition energies, this being specially relevant as the QD complexity, i.e. the number of shells, increases. 

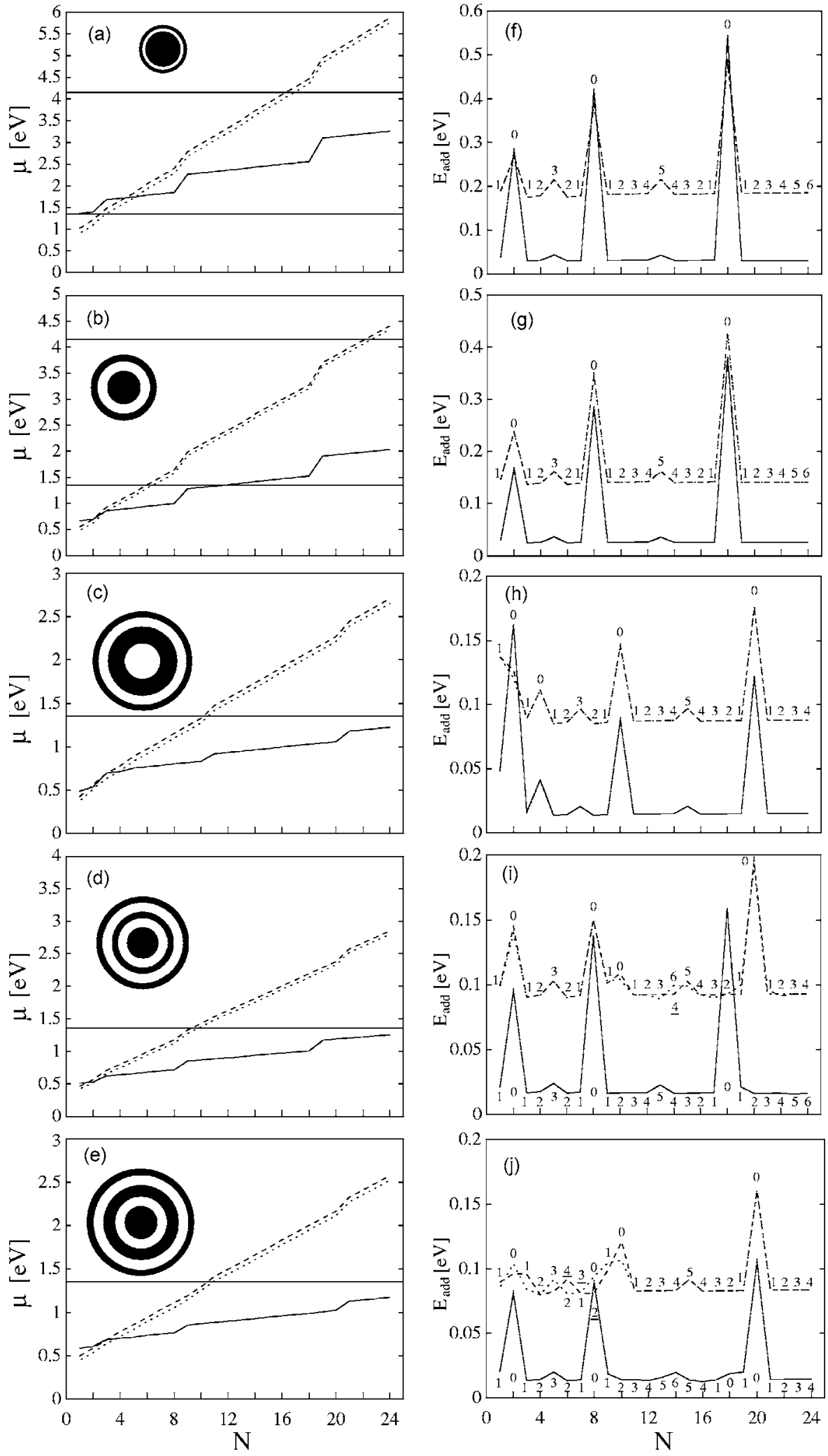

FIG. 1. Chemical potentials (left) and addition energies (right) vs the number $N$ of electrons, corresponding to the QDs outlined in the insets (black for $\mathrm{CdS}$ and white for $\mathrm{HgS}$ ). Solid line: unpolarized calculation. Dotted line: polarized calculation. Dashed line: polarized calculation including self-energy. The horizontal solid lines at 1.35 and $4.15 \mathrm{eV}$ illustrate the confining barrier height for the $\mathrm{HgS}$ well and the QD, respectively. $2 S_{z}$ values are indicated by numbers on the addition spectra. If the spins of the polarized calculation with and without selfenergy are different, underlined numbers correspond to the calculation including self-energy.
We would like to thank Manuel Barranco for useful discussions. Continuous support from MEC-DGI projects CTQ2004-02315/BQU and FIS2005-01414, UJI-
Bancaixa project P1-B2002-01, and Grant No. 2005SGR00343 from Generalitat de Catalunya are gratefully acknowledged. 
${ }^{1}$ A. Eychmüller, A. Mews, and H. Weller, Chem. Phys. Lett. 208, 59 (1993).

${ }^{2}$ A. Mews, A. Eychmüller, M. Giersig, D. Schooss, and H. Weller, J. Phys. Chem. 98, 934 (1994).

${ }^{3}$ M. Braun, S. Link, C. Burda, and M. El-Sayed, Phys. Rev. B 66, 205312 (2002); H. Borchet, D. Dorfs, C. Mc Gingle, S. Adam, T. Möller, H. Weller, and A. Eychmüller, J. Phys. Chem. B 107, 7486 (2003); D. Dorfs, H. Henschel, J. Kolny, and A. Eychmüller, ibid. 108, 1578 (2004); A. Mews, A. V. Kadavanich, U. Banin, and A. P. Alivisatos, Phys. Rev. B 53(20), R13242 (1996).

${ }^{4}$ G. W. Bryant and W. Jaskólski, Phys. Status Solidi B 224, 751 (2001); J. Planelles, J. Climente, J. G. Díaz, and W. Jaskólski, J. Phys.: Condens. Matter 14, 12537 (2002); J. Climente, J. Planelles, J. G. Díaz, and I. Aliaga, ibid. 15, 3539 (2003).

${ }^{5}$ M. Braun, C. Burda, and M. El-Sayed, J. Phys. Chem. A 105, 5548 (2001).

${ }^{6}$ D. Dorfs and A. Eychmüller, Nano Lett. 1(11), 663 (2001); R. Xie, U. Kolb, T. Basché, and A. Mews, J. Am. Chem. Soc. 127, 7480 (2005).

${ }^{7}$ A. Aharoni, T. Mohari, I. Popov, and U. Banin, J. Am. Chem. Soc. 66, 205312 (2006).

${ }^{8}$ J. Pérez Conde and A. K. Bhattacharjee, Phys. Status Solidi B 229, 485 (2002); R. H. Xie, G. W. Bryant, S. Lee, and W. Jaskólski, Phys. Rev. B 65, 235306 (2002); G. W. Bryant and W. Jaskólski, ibid. 67, 205320 (2003).

${ }^{9}$ D. Schooss, A. Mews, A. Eychmüller, and H. Weller, Phys. Rev.
B 49, 17072 (1994); Garnett W. Bryant, ibid. 52, R16997 (1995).

${ }^{10}$ K. Chang and J. B. Xia, Phys. Rev. B 57, 9780 (1998).

${ }^{11}$ W. Jaskólski and Garnett W. Bryant, Phys. Rev. B 57, R4237 (1998).

${ }^{12}$ J. Planelles, J. Díaz, J. Climente, and W. Jaskólski Phys. Rev. B 65, 245302 (2002); J. Planelles, J. Climente, J. Díaz, and W. Jaskólski, J. Phys.: Condens. Matter 14, 12537 (2002); J. Climente, J. Planelles, W. Jaskólski, and J. I. Aliaga, ibid. 15, 3593 (2003).

${ }^{13}$ Y. W. Cao and U. Banin, Angew. Chem., Int. Ed. 38, 3692 (1999).

${ }^{14}$ O. Millo, D. Katz, Y. W. Cao, and U. Banin, Phys. Rev. Lett. 86, 5751 (2001).

${ }^{15}$ Z. Dai, J. Sun, L. Zhang, S. Huang, and J. Zhang, Physica B 324, 373 (2002).

${ }^{16}$ M. Pi, M. Royo, and J. Planelles, J. Appl. Phys. 100, 073712 (2006).

${ }^{17}$ A. D. Yoffe, Adv. Phys. 50, 1 (2001).

${ }^{18}$ U. Von Barth and L. Hedin, J. Phys. C 5, 1629 (1972).

${ }^{19}$ J. L. Movilla and J. Planelles, Comput. Phys. Commun. 170, 144 (2005).

${ }^{20}$ J. P. Perdew and A. Zunger, Phys. Rev. B 23, 5048 (1981).

${ }^{21}$ Chemical potentials and addition energies are calculated as the first and second energy differences, $\mu(i)=E(i+1)-E(i)$, and $E_{\text {add }}(i)=E(i+1)+E(i-1)-2 E(i)$, respectively. 